Groups Geom. Dyn. 7 (2013), 451-474

DOI $10.4171 / \mathrm{GGD} / 190$
Groups, Geometry, and Dynamics

(C) European Mathematical Society

\title{
Scott complexity and adjoining roots to finitely generated groups
}

\author{
Larsen Louder
}

\begin{abstract}
We give several generalizations of the well-known fact that a commutator in a free group is not a proper power.
\end{abstract}

Mathematics Subject Classification (2010). 20F65, 20E06, 20E05, 57M07.

Keywords. Geometric group theory, graphs of groups.

\section{Introduction}

We consider the problem of adjoining roots to elements of finitely generated groups. An element of a free group is primitive if it is part of a basis. The letter $\mathbb{F}$ will be used to represents a nonabelian free group. An element of a group $G$ is indivisible if is not a proper power. Let $G$ be a group. The group $\left\langle G, \gamma_{i}^{\prime} \mid\left(\gamma_{i}^{\prime}\right)^{k_{i}} \gamma_{i}^{-1}\right\rangle$ is denoted $G\left[\sqrt[k]{\gamma_{i}}\right]$.

By [8], if an amalgam $A *\langle t\rangle B$ is finitely generated and free, then $t$ is primitive in one of $A$ or $B$. If $G[\sqrt[k]{\gamma}], k>1$, is free, it corresponds to the case where one of the vertex groups is infinite cyclic, and since $\gamma$ is not primitive in $\langle\sqrt[k]{\gamma}\rangle, \gamma$ is primitive in $G$. The identity map $A *\langle t\rangle B \rightarrow A *\langle t\rangle B$ is a morphism of free groups, and one purpose of this paper is to examine what happens when we are simply given a map $A *\langle t\rangle B \rightarrow \mathbb{F}$ which embeds $A$ and $B$.

Consider Vaught's equation $a^{2} b^{2} c^{2}=1$. Lyndon showed that if $a, b$, and $c$ satisfy Vaught's equation in a free group then they commute [4]. Let $V=\langle a, b, c|$ $\left.a^{2} b^{2} c^{2}\right\rangle$. Solutions to $a^{2} b^{2} c^{2}=1$ in a group $G$ correspond to elements of the set $\operatorname{Hom}(V, G)$, where $V$ is the fundamental group of the connected sum of three projective planes, or equivalently, the connected sum of a torus $T$ and a projective plane $P$. Lyndon's theorem simply says that all elements of $\operatorname{Hom}(V, \mathbb{F})$ factor through the map killing $\partial P$. Equivalently, a commutator in a free group is not a square. Lyndon and Schützenberger [5] (See also [6].) extended this fact to equations $a^{p} b^{q} c^{r}=1$, $p, q, r \geq 2$, of the same type. 
More generally:

Theorem 1.1 ([1]). If $\left(y_{1}, \ldots, y_{n+1}\right)$ is a solution to $\delta\left(x_{1}, \ldots, x_{n}\right)=x_{n+1}^{k}, k>1$, where $\delta$ is a word in $x_{i}$, in $\mathbb{F}$, and $\delta$ is neither a proper power nor primitive in $\left\langle x_{1}, \ldots, x_{n}\right\rangle$, then the subgroup of $\mathbb{F}$ generated by $\left\{y_{1}, \ldots, y_{n+1}\right\}$ has rank less than $n$

The theorems of Lyndon, Lyndon-Schützenberger, and Baumslag, may all be reinterpreted as statements about maps of graphs of free groups to free groups which embed vertex groups.

The purpose of this paper is to generalize this fact to (most) arbitrary finitely generated groups. We define a complexity which reduces to Euler characteristic in the case of a free group, behaves well with respect to adjunctions of roots to arbitrarily many elements, and strictly decreases if roots are added to indivisible (mutually) nonprimitive (collections of) elements. Theorem 1.3 is an important step in the proof that limit groups have finite Krull dimension.

Definition 1.2 ([7], [11]). Let $G$ be a finitely generated group with Grushko decomposition $G_{1} * \cdots * G_{p} * \mathbb{F}_{q}$. The Scott complexity of $G$ is the ordered pair $\chi_{\mathrm{fd}}(G)=(q-1, p)$.

Theorem 1.3. Let $G$ be a finitely generated group, and let $\gamma_{1}, \ldots, \gamma_{n}$ and $\delta_{1}, \ldots, \delta_{m}$ be nontrivial indivisible elements of $G$, such that all $\gamma_{i}$ are contained in freely indecomposable free factors of $G$, no $\delta_{j}$ is contained in any freely indecomposable free factor of $G$, and all $\delta_{j}$ have nonconjugate centralizers. Suppose that $G \hookrightarrow H$ and that the inclusion map extends to a surjection

$$
G^{\prime}=G\left[\sqrt[k_{i}]{\gamma_{i}}, \sqrt[l_{j}]{\delta_{j}}\right] \rightarrow H
$$

Then $\chi_{\mathrm{fd}}(G) \geq \chi_{\mathrm{fd}}(H)$. If equality holds and $H$ has no $\mathbb{Z}_{2}$ free factors then there are elements $d_{j}$ of $G$ such that

$$
G \cong G_{1} * \cdots * G_{p} * F *\left\langle\delta_{1}^{d_{1}}\right\rangle * \cdots *\left\langle\delta_{m}^{d_{m}}\right\rangle
$$

with $G_{i}$ freely indecomposable, and $F$ a free factor.

Theorem 1.1 corresponds to the case where $\chi_{\mathrm{fd}}(G)=\chi_{\mathrm{fd}}(H)=(q-1,0)$, $n=0$, and $m=1$.

Example (No $\mathbb{Z}_{2}$ free factors of $H$ is necessary.). Let $G=A * B$, with $A$ and $B$ freely indecomposable, $H_{1}=A * B /\left\langle\left\langle a b^{-1}\right\rangle, a \in A \backslash 1, b \in B \backslash 1\right.$. Let $H=H_{1} *\left\langle x \mid x^{2}\right\rangle$. Suppose that the natural maps $A, B \hookrightarrow H_{1}$ are injective. Then the map $G \rightarrow H$ which sends $A$ to $A<H_{1}$ and $B$ to $x B x^{-1}<x H_{1} x^{-1}$ is injective, $a \mapsto a, b \mapsto x b x^{-1}=x a x$, and $a b \mapsto(a x)^{2}$. Then $H$ is a quotient of $G[\sqrt{a b}]$ but $\chi_{\mathrm{fd}}(G)=\chi_{\mathrm{fd}}(H)=(0,2)$. 
Our analysis also gives the following two theorems, which we prove in Section 2.4.

Theorem 1.4 (Improved Shenitzer's Theorem). Let $G=A *\langle t\rangle B, A$ and $B$ free of rank $n$ and $m$, respectively. If $t$ is indivisible in at least one of $A$ or $B$ and $G$ maps onto a free group of rank $n+m-1$, then $G$ is free and $t$ is primitive in at least one of $A$ or $B$. If $t$ is not indivisible in either factor then $G$ is a free product of a free group and an amalgamated product of two infinite cyclic groups.

Baumslag obliquely suggests a conjugacy separability problem [1], pp. 278-279, which we interpret as:

Theorem 1.5. Let $F$ be a free group of rank $n>1$, and let $\left\{t_{i}\right\}$ be stable letters, and let $\left\{\delta_{j}\right\}$ be nontrivial, nonconjugate elements of $F$. Let $G$ be the group $F *$ $\left.\left\langle t_{i}\right\rangle /\left\langle t_{i} \delta_{j_{i}} t_{i}^{-1}=\delta_{j_{i}^{\prime}}\right\rangle\right\rangle$, and assume that each $\delta_{j}$ participates. If $G$ has a map onto a free group of rank $n$ embedding $F$, then $F \cong F^{\prime} *\left\langle\delta_{j}\right\rangle$ for some $j$, with each element $\gamma_{j^{\prime}}, j^{\prime} \neq j$, conjugate (in $F$ ) into $F^{\prime}$.

Acknowledgments. It is hard to overstate my gratitude to Mark Feighn for his careful reading of more versions of this paper than I can count. I would also like to thank Mladen Bestvina for telling me the proof of Shenitzer's theorem and teaching me folding. I would also like to thank the referee.

The author was supported in part by EPSRC grant EP/D073626/2 and NSF MSPRF DMS-0703658.

\section{Free groups}

2.1. Immersions I. A graph is a one dimensional $\mathrm{CW}$ complex. A graph with boundary $\Gamma$ is a graph with some valence one vertices marked as elements of the boundary $\partial \Gamma$. A graph is a core graph of it has no valence one vertices outside its boundary. A morphism of graphs is a map that sends vertices to vertices and maps interiors of edges homeomorphically to interiors of edges. A morphism of graphs is an immersion if it is locally injective. Equivalently, a morphism of graphs is an immersion if it is injective on links of vertices. Stallings observed that if $f: A \hookrightarrow B$ is a morphism of finitely generated free groups then there is an immersion of finite graphs $\varphi: \Gamma_{A} \rightarrow \Gamma_{B}$ such that after identifying $A$ and $B$ with the fundamental groups of $\Gamma_{A}$ and $\Gamma_{B}$ appropriately, $\varphi$ represents $f$. If $\Gamma$ is a graph and $p$ is a closed path crossing some edge of $\Gamma$ exactly once then $p$ represents a primitive element in $\pi_{1}(\Gamma)$.

Let $b$ and $r$ be circles, and let $l: b \rightarrow r$ be the $l$-fold cover. The mapping cylinder $M(l)$ of $l$ is the space $b \times[0,1] \sqcup r /(x, 1) \sim l(x)$. We call $r \subset M(l)$ the core, and we call $b \times\{0\} \subset M(l)$ the boundary. Let $\Gamma$ be a graph, and let $\gamma: S^{1} \rightarrow \Gamma$ be a cyclically reduced path, and suppose that $\gamma$ has an $l$-th root, that is, a cyclically reduced path $\sqrt[l]{\gamma}: S^{1} \rightarrow \Gamma$ such that $(\sqrt[l]{\gamma})^{l}$ is homotopic to $\gamma$ through reduced edge 
paths. By the mapping cylinder $M(\gamma, l)$ we mean the mapping cylinder of the $l$-fold cover of $S^{1}$, subdivided into squares, such that the edges of squares not contained in the boundary or core are sent to vertices, and the restrictions to $b \times\{x\}$ are reduced edge paths, and with the natural map $M(\gamma, l) \rightarrow \Gamma$ induced by $\gamma, \sqrt[l]{\gamma}$, and the homotopy. See Figure 1.

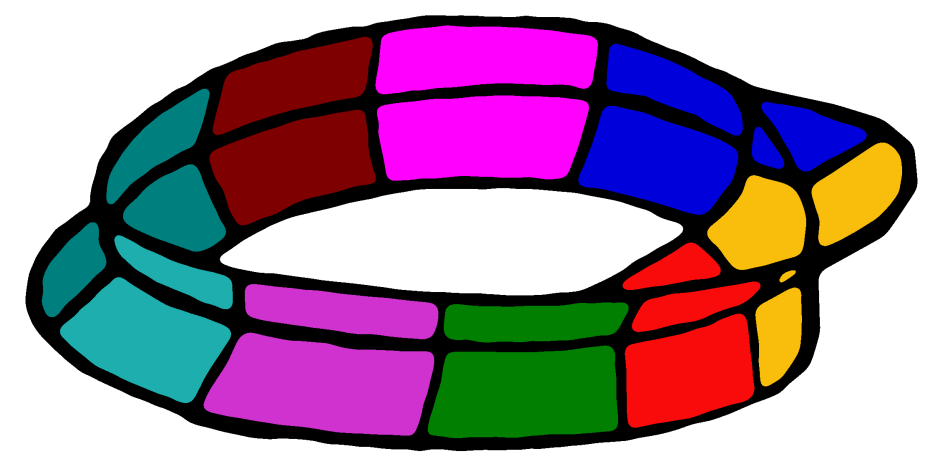

Figure 1. A subdivided mapping cylinder $S^{1} \stackrel{\times 3}{\longrightarrow} S^{1}$.

To motivate the approach to Theorem 1.3 we first give a proof of Shenitzer's Theorem: If $G=A *\langle t\rangle B$ is free, then the inclusion maps $A, B \hookrightarrow G$ may be represented by immersions $\Gamma_{A} \rightarrow R, \Gamma_{B} \rightarrow R$, where $R$ is a graph with fundamental group identified with $G$. Represent $\langle t\rangle$ by a pair of immersions $S^{1} \rightarrow \Gamma_{A}, \Gamma_{B}$ and glue the boundary components of an (subdivided) annulus to $\Gamma_{A} \sqcup \Gamma_{B}$ along the immersions of $S^{1}$ to obtain a graph of spaces $X$. Extend $\Gamma_{A}, \Gamma_{B} \rightarrow R$ to a map $\varphi: X \rightarrow R$. Pull back midpoints of edges of $R$ to produce embedded graphs in $X$ "transverse" to both $\Gamma_{A}$ and $\Gamma_{B}$. The hypothesis that $\Gamma_{A}, \Gamma_{B} \rightarrow R$ are immersions implies that the preimage graphs must be finite forests, otherwise the identity map $G \rightarrow G$ has nontrivial kernel. This implies that the representative of $t$ as an immersion, in at least one of $\Gamma_{A}$ or $\Gamma_{B}$, must cover some edge exactly once, and $t$ is therefore a basis element in one of $A$ or $B$.

Consider Theorem 1.1: A solution to $\delta\left(x_{1}, \ldots, x_{n}\right)=x_{n+1}^{k}, \delta$ a word in $x_{i}$ and $k>1$, in a free group $\mathbb{F}$ corresponds to a map $\left\langle x_{1}, \ldots, x_{n}\right\rangle[\sqrt[k]{\delta}] \rightarrow \mathbb{F}$. If the image of $\left\langle x_{1}, \ldots, x_{n}\right\rangle$ in $\mathbb{F}$ has rank less than $n$ then the conclusion holds, so we may assume that the map is an embedding on $\left\langle x_{i}\right\rangle$. The group $\left\langle x_{1}, \ldots, x_{n}\right\rangle[\sqrt[k]{\delta}]$ is an amalgam of a free group and a cyclic group. We construct a space $X$ and a map $X \rightarrow R$ representing the map $\left\langle x_{1}, \ldots, x_{n}\right\rangle[\sqrt[k]{\delta}] \rightarrow \mathbb{F}$ exactly as in the proof of Shenitzer's theorem. The main observation of this paper is that if the image of $\left\langle x_{1}, \ldots, x_{n}\right\rangle[\sqrt[k]{\delta}]$ has rank $n$ then the preimage graphs are forestlike, and if $k>0$ and $\delta$ is indivisible, then they are indeed forests. Using this and the fact that a closed path crossing some edge only once represents a primitive element we conclude again that $\delta$ must be primitive. In the following subsection we make this argument precise. 
2.2. Graphs of graphs I. The following is from [3]. A graph of graphs is a graph of spaces such that all vertex and edge spaces are finite combinatorial graphs and all edge maps are embeddings. A graph is 2-covered by a family of subgraphs if every edge is contained in exactly two elements of the cover, and a graph of graphs $X$ is 2-covered if each vertex space is 2-covered by incident edge spaces. A graph of graphs $X$ has an underlying graph $\Gamma_{U}(X)$, and corresponds to a map from $\Gamma_{U}(X)$ to the category of combinatorial graphs and injective maps. We define the topological realization of $X$ in the usual way: For each edge space $E$ introduce a product $E \times I$ and identify the points in $E \times\{0\}$ and $E \times\{1\}$ with their images in vertex spaces under the edge maps associated to $E$. We will usually conflate graphs of spaces with their topological realizations. A 2-covered graph of graphs with boundary is a graph of graphs such that non-valence-one vertex spaces are 2-covered by incident edge spaces, and valence one vertex spaces are isomorphic to their incident edge space. An edge of a vertex or edge space is vertical.

Let $\Delta\left(A_{i}, B_{j}\right)$ be shorthand for a graph of groups with underlying graph $\Delta$, vertex groups $A_{i}$, and edge groups $B_{j}$. Let $G=\Delta\left(F_{i}, Z_{j}\right)$ be a graph of free groups $F_{i}$ over nontrivial subgroups $Z_{j}$. If $\phi: G \rightarrow \mathbb{F}$ is a homomorphism embedding each $F_{i}<G$, then there is a graph of spaces with fundamental group $G$ and a natural map to a graph representing $\phi$. For each $i$, choose an immersion $\varphi_{i}: \Gamma_{i} \rightarrow R$ where $R$ is the rose with $n$ petals, and fundamental group $\mathbb{F}$ and $\pi_{1}\left(\Gamma_{i}\right)=F_{i}$, and $\Gamma_{i}$ a core graph. For each $j$, choose an immersion $\varphi_{j}: \Delta_{j} \rightarrow R_{n}$ representing the image of $Z_{j}$. If $Z_{j} \hookrightarrow F_{i}$ then $\varphi_{j}$ lifts to an immersion $\varphi_{i, j}: \Delta_{j} \rightarrow \Gamma_{i}$. Use the $\varphi_{i, j}$ as attaching maps of annuli, one for each edge of $\Delta$, to define a graph of spaces $X$. The homomorphism $\phi$ induces a map $\varphi: X \rightarrow R$. Restricted to a product $\Delta_{j} \times \mathrm{I}$, the map is projection to the first factor, followed by the immersion $\varphi_{j}$. Let $b$ be the basepoint of $R$.

Let $\left\{V_{p}\right\}$ be the collection of connected components of $\varphi^{-1}(b)$, let edge graphs be connected components of preimages of midpoints of edges of $R$ and regard $X$ as a graph of graphs by regarding the $V_{p}$ as vertex graphs. The homomorphism $F_{i} \hookrightarrow G$ is represented by the inclusion $\Gamma_{i} \rightarrow X$, and the map $X \rightarrow R$ therefore represents the map $G \rightarrow \mathbb{F}$. Given $\phi: G \rightarrow \mathbb{F}$ as above, we sometimes write $X(G, \phi)$ to denote $X$. Since all vertical edges come from annuli $X$ is 2-covered.

Theorem 2.1. Let $\pi: G\left[\sqrt[l]{\delta_{j}}\right] \rightarrow H$ be as in Theorem 1.3, and suppose further that $G$ and $H$ are free. Then $\operatorname{rk}(H) \leq \operatorname{rk}(G)$. If equality holds then the edge spaces of $X\left(G\left[\sqrt[l_{i}]{\delta_{j}}\right], \pi\right)$ are forests.

We postpone the proof of Theorem 2.1 until Section 2.4.

The horizontal subgraph $\Gamma(X)$ of a graph of graphs $X$ is the subset of the topological realization of $X$ obtained by restricting to the zero skeleta of the vertex and edge spaces. Let $\Gamma_{\infty}(X)$ be the subset of $\Gamma(X)$ consisting of the connected components not homeomorphic to $S^{1}$. For $X(G, \phi)$ as above, $\Gamma_{\infty}(X)=\bigcup_{i} \Gamma_{i} \subset X$. We assume throughout that if $X$ is a graph of graphs without boundary, and $e$ is an edge of a 
vertex space, then $e$ is covered by at least two edges from incident edge spaces. This is equivalent to saying that the graph obtained by restricting to midpoints of edges of vertex and edge spaces is a core graph.

2.3. Blowing up. Let $X$ be a graph of graphs. If $e$ is an edge in the underlying graph of $X$ then $X / e$, the graph of graphs obtained by blowing-down $X$ is obtained by collapsing the edge space $E \times I$ in the topological realization of $X$ to $E$, provided the quotient map $X \rightarrow X / e$ is a homotopy equivalence. The inverse of blowing down is blowing up.

Theorem 2.2 ([3], Theorem 2.4). Given a graph of graphs $X$ there is a homotopy equivalent graph of graphs $Y$, obtained by repeatedly blowing up, such that each edge space of $X$ is homeomorphic to a disjoint union of edge spaces of $Y$, vertex and edge spaces of $Y$ are connected, and each vertex space of $Y$ has exactly three incident edge spaces.

Lemma 2.3 (Euler Characteristic Lemma). Let $X$ be a graph of graphs with connected vertex and edge spaces. Then

$$
\chi(\Gamma(X)) \leq \chi\left(\Gamma_{U}(X)\right) .
$$

Suppose that $\chi(\Gamma(X))=\chi\left(\Gamma_{U}(X)\right)$ and every vertex space has three incident edge spaces. If $E_{1}, E_{2}$ and $E_{3}$ are incident to a vertex space $V$ with no monogons then $E_{1} \cap E_{2} \cap E_{3}$ is a single point and $V$ is 2-covered.

Proof. Identical to the proof of Theorem 1.1 of [3]. Blow $X$ up to a homotopy equivalent space $Y$ with only valence three vertex spaces. Blowing up and down do not change the Euler characteristic of the horizontal graph. If $V$ is a vertex space with three incident edges, connectivity implies that there is a vertex of $V$ in the image of all three incident edge spaces. This vertex corresponds to a valence three vertex of $\Gamma(X)$, therefore the map from valence three vertices of $\Gamma(X)$ to vertex spaces of $X$ is surjective. Then by combinatorial Gauss-Bonnet $\chi(\Gamma(X)) \leq \chi\left(\Gamma_{U}(X)\right)$.

If $\chi(\Gamma(X))=\chi\left(\Gamma_{U}(X)\right)$ the map from valence three vertices of the horizontal graph to the set of valence three vertices of the underlying graph is bijective. If an edge of a vertex space is covered three times by incident edge spaces then the endpoints are covered three times by incident edge spaces. Since the valence three vertices of the underlying graph and the horizontal graph are in bijective correspondence, the endpoints of the offending edge must be the same, hence $V$ contains a monogon.

Graphs of spaces whose horizontal subgraphs have the same Euler characteristic as their underlying graphs are particularly important. In this case vertex spaces have a simple local structure.

Lemma 2.4. A graph $V$, 2-covered by connected subgraphs $E_{i} \subset V, i=1,2,3$, such that $\bigcap \operatorname{Im}\left(E_{i}\right)$ is a single vertex $w$, has one of the following forms: 
- $E_{i}$ are all points.

- $E_{1}$ is a point, and $E_{2 \mid 3} \cong V$.

- $V=\operatorname{Im}\left(E_{1}\right) \vee_{w} \operatorname{Im}\left(E_{2}\right), E_{3} \cong V$.

- $V$ is the union of three subgraphs $V_{1|2| 3}$ which meet at a single vertex $w \in V \cong$ $\bigvee_{w} V_{i} . E_{i} \cong V_{i+1} \vee_{w} V_{i+2}$.

If $V$ is as above and $W \subset V$ we say that $W$ is cut by $w$ if $W$ is not contained in some $V_{i}$. If $W_{1}, W_{2} \subset V$ and neither $W_{1}$ nor $W_{2}$ is cut by $w$ then we say that $w$ separates $W_{1}$ and $W_{2}$ if, without loss of generality, $W_{1} \subset V_{1}, W_{1} \not \subset V_{2}$, and $W_{2} \subset V_{2}$, $W_{2} \not \subset V_{1}$.

Proof of Lemma 2.4. Let $\mathcal{P}$ be the set of edge-paths starting at $w$ and terminating if they meet $w$ again. Let $\left\{E_{i}\right\}_{i=0,1,2}$ be the edge graphs incident to $V$. We divide $\mathcal{P}$ into three subclasses $\mathcal{P}_{j, k}, j \neq k$. A path $p$ lies in $\mathcal{P}_{j, k}$ if the image of $p$ is contained in $\operatorname{Im}\left(E_{j}\right) \cap \operatorname{Im}\left(E_{k}\right)$. Let $V_{i}=\bigcup_{p \in \mathcal{P}_{i+1, i+2}} \operatorname{Im}(p)$. At most one of $V_{i}$ can be a point. Every point/edge of $V$ lies in one of $V_{i}$, which all meet at $w$, the sole point of triple intersection. An incident edge $E_{i}$ is then isomorphic to $V_{i+1} \cup V_{i+2}$.

2.4. Cylinders. A morphism of graphs of graphs is a morphism of graphs of spaces immersing vertex and edge spaces, respecting the product structure of edge spaces, in vertex and edge spaces, respectively. A cylinder in a graph of spaces $X$ is a morphism $C \rightarrow X$, where $C$ is a graph of graphs with all edge maps isomorphisms of connected graphs and with underlying graph a circle. Furthermore, the induced map $\Gamma_{U}(C) \rightarrow \Gamma_{U}(X)$ should be a cyclically reduced path. Cylinders are partially ordered by the relation "factors through", i.e., $C \geq C^{\prime}$ if $C^{\prime} \rightarrow C \rightarrow X$.

Let $C \rightarrow X$ be a cylinder, and let $F$ be a vertex space of $C$. If the map $F \rightarrow X$ is not an immersion, there are oriented edges $h$ and $k$ with the same initial endpoint with the same image in a vertex graph of $X$. Let $E \rightarrow F$ be an incident edge map. The edges $e$ and $f$ are the images of edges $h^{\prime}$ and $k^{\prime}$ in $E$. Since edge maps of $C$ are all isomorphisms of graphs there is a rotation map $C \rightarrow C$ given by translating each vertex and edge space one unit: If edges are ordered coherently and $\left(\iota_{i}\right)$ and $\left(\tau_{i}\right)$ are the edge maps then the rotation map is given by $\tau_{i} l_{i}^{-1}$ on vertex spaces and by $\iota_{i+1}^{-1} \tau_{i}$ on edge spaces. If $(a, b)$ is an element of the orbit of $\left(h^{(\prime)}, k^{(\prime)}\right)$ then $a$ and $b$ are folded together by the associated map $C \rightarrow X$. Fold all pairs in the orbits of $\left(h^{(\prime)}, k^{(\prime)}\right)$ to obtain a new cylinder $C^{\prime} \rightarrow X$. The map $C \rightarrow X$ factors through the natural map $C^{\prime} \rightarrow X$. Since folding is equivariant with respect to rotation $C^{\prime} \rightarrow X$ is a cylinder.

Let $f_{i}: C_{i} \rightarrow X$ be a collection of cylinders. As in the case of a graph, we define the pullback of $\left\{C_{i}\right\}$ to be the graph of graphs

$$
P=\left\{\left(x_{1}, \ldots, x_{n}\right) \in C_{1} \times \cdots \times C_{n} \mid f_{i}\left(x_{i}\right)=f_{j}\left(x_{j}\right)\right\} .
$$

There are induced maps $g_{i}: P \rightarrow C_{i}$. If $C_{1}, C_{2} \rightarrow X$ are cylinders and their pullback contains a cylinder then either, without loss, $C_{1} \geq C_{2}$ or neither is maximal. Suppose 
that $Z$ is a cylindrical component of the pullback. Then $C^{\prime}=\left(C_{1} \cup C_{2} \cup Z\right) /\left(g_{1}(z) \sim\right.$ $\left.g_{2}(z)\right)$ is a cylinder, and has an induced map $C^{\prime} \rightarrow X$. Now fold to obtain a larger cylinder $C^{\prime \prime} \rightarrow X$. If $C \rightarrow X$ is a cylinder, consider the (self) pullback of the collection $C, C \rightarrow X$ : if it contains a cylinder other than the diagonal then $C$ is not maximal.

Let $X$ be a graph of graphs, let $I$ be a subdivided interval, and let $p: I \rightarrow$ $X$ be a reduced edge path in $\Gamma(X)$. Let $V$ be a vertex space of $X$, and say that $\eta: F \rightarrow V \subset X$ pushes along $p$ if $p(0)=\eta(v)$ for some vertex $v$ of $F$ and the map $(I \cup F) /(0 \sim v) \rightarrow X$ extends to the product $I \times F$ and the reduced edge paths obtained by restricting to vertices of $F$ are all identical.

Lemma 2.5. Suppose that $\chi(\Gamma(X))=\chi\left(\Gamma_{U}(X)\right), \eta: F \rightarrow X$ pushes along $p$ and $p^{\prime}$, the first edges of the projections of $p$ and $p^{\prime}$ to $\Gamma_{U}(X)$ agree, and that $F$ has more than one point. Then, without loss of generality, the projection of $p$ to $\Gamma_{U}(X)$ is a sub-path of the projection of $p^{\prime}$ to $\Gamma_{U}(X)$.

Proof. Since $F$ has more than one point, images of vertex spaces of $I \times F$ are uncut by valence three vertices of $\Gamma(X)$. We may assume by removing prefixes that the projections of $p$ and $p^{\prime}$ diverge after one step, and that they are both of length two, but this implies that the image of $F$ in the vertex space where the two paths diverge is cut by a valence three vertex of $\Gamma(X)$, contradicting the fact that $F$ pushes past that vertex space.

Lemma 2.6. Suppose that $\chi(\Gamma(X))=\chi\left(\Gamma_{U}(X)\right)$. Suppose that $\eta$ : $F \rightarrow X$ pushes along a path $p$, and suppose $\eta(x)=\eta(y)$ for some $x \neq y$. Let $F^{\prime} \rightarrow X$ be the immersed graph obtained by identifying $x$ and $y$ and folding. Then $F^{\prime}$ pushes along $p$.

Proof. The two element set $\{x, y\}$ pushes along $p$, and $F / x \sim y$ therefore pushes along $p$. Furthermore, any two edges of $F /(x \sim y)$ adjacent to $[x]$ push along $p$, and since $X$ is 2-covered, they push in a unique way, hence if $e$ and $f$ are identified by folding, then $(F / x \sim y) /(e \sim f)$ pushes along $p$. The lemma follows by induction on the number of edges.

Lemma 2.7 (Vertex spaces embed). Let $X$ be a 2-covered graph of spaces and let $C \rightarrow X$ be a cylinder. If $E$ is an edge space of $C$ and the induced map $E \rightarrow X$ is not injective then $C$ is not maximal.

Proof. Identify points having the same images and fold.

Lemma 2.8 (Vertex spaces do not overlap much). Let $C, C^{\prime} \rightarrow X$ be two cylinders such that all maps of vertex spaces are embeddings, and suppose two vertex spaces $E$ and $E^{\prime}$ of $C$ and $C^{\prime}$, respectively, intersect in more than one point. Then either (without loss of generality) $C \geq C^{\prime}$ or neither is maximal. 
Proof. The multi-element set $E \cap E^{\prime} \subset X$ pushes along arbitrarily long reduced edge paths, but the pullback of $C$ and $C^{\prime}$ is finite, therefore there is a cylindrical component of the pullback.

Existence of maximal cylinders is guaranteed by the previous corollaries. Let $Y$ be obtained from $X$ by blowing up. If $C$ is a maximal cylinder in $X$ then there is a maximal cylinder $D$ in $Y$ such that $C$ is a blow-down of $D$ and the two maps $D \rightarrow Y \rightarrow X$ and $D \rightarrow C \rightarrow X$ agree. In particular, the collection of types of cylinder cross-sections of $X$ and $Y$ are the same.

Lemma 2.9 (No cycles). Let $V$ be a vertex space of $X$, and suppose that $C_{0}, \ldots, C_{n-1}$ are distinct maximal cylinders, $E_{0}, \ldots, E_{n-1}$ vertex spaces of $C_{i}$, such that $C_{i} \rightarrow X$ embeds $E_{i}$ in $V$. If $E_{(i \bmod n)} \cap E_{i-1} \neq \emptyset$ then $E_{i} \cap E_{j} \neq \emptyset$ for some $|i-j|>1$. Intersections of images of edge graphs of maximal cylinders are cut-points in the associated edge spaces of $X$.

Proof. Suppose not. Without loss of generality there are paths $p_{i, i+1}, i=0, \ldots, n-2$, such that $E_{i} \cap E_{i+1}$ pushes along $p_{i, i+1}$, the projection $p$ of $p_{i, i+1}$ to $\Gamma_{U}(X)$ agree, and $E_{0} \cap E_{n-1}$ does not push along any path projecting to $p$. Choose a longest path $p^{\prime}$ projecting to a sub-path of $p$ that $E_{0} \cap E_{n-1}$ pushes along. Then the images of $E_{0}$ and $E_{n-1}$ are separated by the valence three vertex of the vertex space corresponding to the end of $p$ ', but the relation 'not separated by' is transitive. Thus the sets $E_{i} \cap E_{i+1}$ push along arbitrarily long paths, and the pullback of $C_{i}$ and $C_{i+1}$ must contain a cylinder.
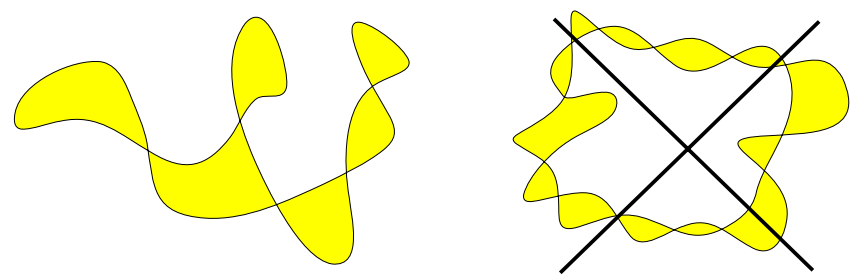

Figure 2. Edge spaces are trees of cylinder cross-sections. The shaded regions are cylinder cross-sections. On the right there is a cycle, which does not occur, and on the left the crosssections are glued in a treelike pattern.

Proof of Theorem 2.1. Consider the graph of graphs $X=X\left(G\left[l_{i} \sqrt{\delta_{j}}\right], \phi\right)$. Blow $X$ up to a graph of graphs $Y$ with only valence three vertices. Each edge space of $X$ is a disjoint union of edge spaces of $Y$. We show that the edge spaces of $Y$ are trees. Assuming $\operatorname{rk}(G)=\operatorname{rk}(H)$, we have $\chi(\Gamma(Y))=\chi\left(\Gamma_{U}(Y)\right)$. Let $M_{j}\left(\delta_{j}, l_{j}\right)$ be the subdivided mapping cylinder corresponding to $\delta_{j}$, now regarded as an element of $\Gamma_{\infty}(Y)$. Then $Y$ is obtained by gluing the boundaries of the mapping cylinders 
$M_{j}$ to $\Gamma_{\infty}(Y)$ along reduced edge paths representing the $\delta_{j}$. The map $M_{j} \rightarrow Y$ is a cylinder, and factors through a maximal cylinder $C$. Each $\delta_{j}$ is indivisible, therefore edge graphs of $M_{j}$ embed in $Y$, hence edge graphs of $M_{j}$ embed in $C$, and since no two $M_{j}$ are conjugate or overlap in a vertical edge, $C$ is the union of some subcollection of the $M_{j}$. Since the $\delta_{j}$ are nonconjugate, $C=M_{j}$ for some $j$. By Lemma 2.9 intersections of images of edge graphs of the $M_{j}$ are cut points of edge spaces of $Y$. If $e$ is an (vertical) edge of an edge space of $Y$ then $e$ is contained in the image of some cross section of some $M_{j}$, and therefore each nontrivial edge space of $Y$ is a union of trees along cut-points, hence is a tree.

Proof of Theorem 1.3 when $G$ and $H$ are free. By Theorem 2.1 edge spaces of $X$ are forests, and since $l_{j}>0$ there is some element $\delta_{j}$ which crosses an edge $f$ of $\Gamma_{\infty}(X)$ only once, and no other $\delta_{j^{\prime}}$ crosses $f$. Thus $G$ can be written as $F *\left\langle\delta_{j}\right\rangle$ with all $\delta_{j^{\prime}}, j^{\prime} \neq j$, conjugate into $F$. Induct.

Proof of Theorem 1.4. The proof is exactly the same as the previous proof. Indivisibility of $t$ in at least one factor implies that edge spaces of $X(G)$ are forests, and a valence one vertex of a forest corresponds to an edge that an immersion representing $t$ in one of $A$ or $B$ crosses exactly once, hence $t$ is primitive in at least one factor.

Proof of Theorem 1.5. The hypotheses are again designed to imply that edge spaces of $X(G, \phi)$ are forests.

\section{Groups}

3.1. Immersions II. Immersions of (core) graphs efficiently represent inclusions of free groups. We mimic this and represent injections

$$
G=G_{1} * \cdots * G_{p_{G}} * \mathbb{F}_{q_{G}} \hookrightarrow H=H_{1} * \cdots * H_{p_{H}} * \mathbb{F}_{q_{H}}
$$

with immersions of graphlike complexes.

Definition 3.1 (Relative graph). A relative graph is a CW-complex $\Gamma$ equipped with a pair of collections of disjoint connected subcomplexes

$$
\operatorname{Ell}(\Gamma)=\left\{Y_{1}, \ldots, Y_{p}\right\} \subset \operatorname{Verts}(\Gamma)=\left\{V_{1}, \ldots, V_{b}\right\}
$$

such that cells not contained in any $Y \in \operatorname{Verts}(\Gamma)$ are one-dimensional, and if $\pi_{1}(Y) \neq 1, Y \in \operatorname{Verts}(\Gamma)$, then $Y \in \operatorname{Ell}(\Gamma)$.

The vertices of a relative graph are the elements of $\operatorname{Verts}(\Gamma)$. The edges of a relative graph are the one-cells not contained in any element of $\operatorname{Verts}(\Gamma)$. The valence of a vertex $v$ is the number of oriented edges with endpoints in $v$. A vertex is a tree if it has trivial fundamental group, and is empty if it is just a 0-cell. A relative graph with boundary is a relative graph with some valence one empty vertices marked as 
elements of $\partial \Gamma$. A relative graph $\Gamma$ is reduced if all valence one empty vertices are in $\partial \Gamma$.

Definition 3.2 (Morphism of relative graphs). Let $\Gamma$ and $\Gamma^{\prime}$ be relative graphs. A continuous map $\varphi: \Gamma \rightarrow \Gamma^{\prime}$ is a morphism of relative graphs if elements of $\operatorname{Verts}(\Gamma)$ are mapped to elements of Verts $\left(\Gamma^{\prime}\right)$, and interiors of edges of $\Gamma$ are mapped homeomorphically to interiors of edges of $\Gamma^{\prime}$.

Denote by $\Gamma / \operatorname{Verts}(\Gamma)$ the (ordinary) graph obtained by collapsing all elements of $\operatorname{Verts}(\Gamma)$ to points.

Definition 3.3. The complexity of a relative graph is the pair

$$
\chi_{\mathrm{fd}}(\Gamma):=(-\chi(\Gamma / \operatorname{Verts}(\Gamma)),|\operatorname{Ell}(\Gamma)|) .
$$

Pick a (empty or nonempty) vertex $v$. Take a copy of $v$, and for each oriented edge $e$ of $\Gamma$ with terminal endpoint in $v$ attach a copy of $e$ at the corresponding point to obtain the star of $v, \operatorname{St}(v)$. There is a natural map $\operatorname{St}(v) \rightarrow \Gamma$.

Definition 3.4 (Immersion/binding ties [9]). Let $\varphi: \Gamma^{\prime} \rightarrow \Gamma$ be a morphism of relative graphs. We say that $\varphi$ is an immersion if, for each vertex $v$ of $\Gamma$ and each connected components $w$ of $\varphi^{-1}(v)$,

$$
\pi_{1}\left(\varphi_{w}\right): \pi_{1}(\operatorname{St}(w), \partial \operatorname{St}(w)) \rightarrow \pi_{1}(\operatorname{St}(v), \partial \operatorname{St}(v))
$$

is injective.

Let $\varphi: \Gamma \rightarrow \Gamma^{\prime}$ be a morphism of graphs. We say that $\varphi$ is a Stallings fold if $\varphi$ simply identifies a pair of edges having a common endpoint. Any morphism of graphs factors through a sequence of Stallings folds followed by an immersion. We define a family of moves on relative graphs which are inspired by Dunwoody's folding sequences [2].

- Enlarging a subcomplex: Replace an element of $\operatorname{Verts}(\Gamma)$ by a larger subcomplex of $\Gamma$.

- Collapsing to a point: Replace $Y \in \operatorname{Verts}(\Gamma)$ by a point.

- If $v$ is a tree vertex of $\Gamma$ and $\Gamma \rightarrow \Gamma^{\prime}$ is a morphism of relative graphs, let $b_{1}, \ldots, b_{n}$ be the 0 -cells of $\Gamma$ contained in $v$ which intersect an edge of $\Gamma$. Let $v^{\prime}$ be a reduced tree with $n$ boundary components $b_{1}^{\prime}, \ldots, b_{n}^{\prime}$, and let $\Gamma^{\prime \prime}$ be the relative graph obtained by replacing $v$ by $v^{\prime}$, gluing the edges of $\Gamma$ attached to $v$ at $b_{i}$ to $b_{i}^{\prime}$. There are maps $\Gamma^{\prime \prime} \rightarrow \Gamma \rightarrow \Gamma^{\prime}$ such that $\Gamma^{\prime \prime} \rightarrow \Gamma^{\prime}$ is the identity away from $v$ and $v^{\prime}$, and an isomorphism of fundamental groups.

- Attaching 2-cells: Let $\Gamma$ be a relative graph. If $Y \in \operatorname{Ell}(\Gamma)$ attach 2-cells to $Y$. 
- Folding: See Figure 3. Let $\Gamma$ be a relative graph, let $v$ be a vertex of $\Gamma$, and let $e$ and $f$ be distinct edges with terminal endpoints in $v$. Let $D$ be a square, and attach three consecutive sides of $D$ to $\Gamma$ by a path $e p \bar{f}$, where $p$ is a path in $v$. (Recall that $v$ may have nontrivial fundamental group.) Collapse $D$ by collapsing the face attached to $f$ to the remaining three sides, and if the fourth side of $D$ connects two 0 -cells of $\Gamma$ crush the fourth side to a point to obtain $\Gamma^{\prime}$. The vertices of $\Gamma^{\prime}$ are obtained from those of $\Gamma$ by taking connected components of the image of $\operatorname{Verts}(\Gamma)$ and the fourth side of $D$. If $v$ is an ordinary vertex then the path $p$ is trivial and a fold is just a Stallings fold. If either $w$ or $w^{\prime}$ is in $\operatorname{Ell}(\Gamma)$, or if $w=w^{\prime}$, we add $w \cup h \cup w^{\prime}$ to $\operatorname{Ell}\left(\Gamma^{\prime}\right)$.

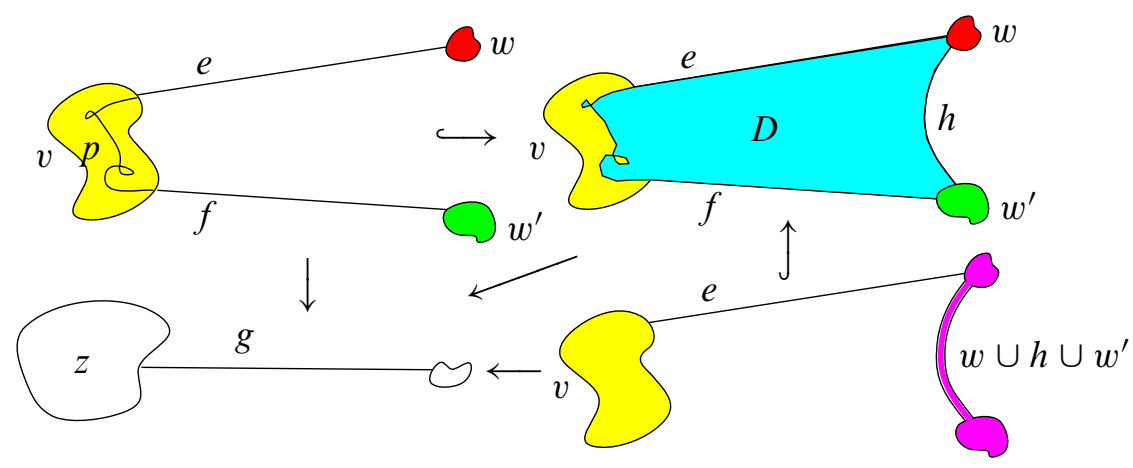

Figure 3. Folding.

The following lemma is the analogue for relative graphs of Stallings' theorem that morphisms of graphs factor through sequences of Stallings folds. The way $\chi_{\mathrm{fd}}$ behaves is similar to the complexity used in Weidmann's proof of acylindrical accessibility [12].

Lemma 3.5 (cf. [10]). If $\varphi: \Gamma \rightarrow \Gamma^{\prime}$ is combinatorial, then there is a relative graph $\bar{\Gamma}, \chi_{\mathrm{fd}}(\bar{\Gamma}) \leq \chi_{\mathrm{fd}}(\Gamma)$, a $\pi_{1}$-onto map $F: \Gamma \rightarrow \bar{\Gamma}$, and an immersion $\bar{\varphi}: \bar{\Gamma} \rightarrow \Gamma^{\prime}$, such that $\bar{\varphi} \circ F$ is homotopic to $\varphi$, and $F$ is a composition of folds. Immersions are injective on fundamental groups.

The proof of Lemma 3.5 is an easy variation on Stallings/Dunwoody folding sequences.

Proof. If, at any point, $\varphi$ maps a nonempty vertex to a point, collapse the vertex to a point. The complexity strictly decreases if the vertex is in $\operatorname{Ell}(\Gamma)$. More generally, if $\varphi$ is not injective on $\pi_{1}(Y)$ for some $Y \in \operatorname{Ell}(\Gamma)$ attach enough two-cells to $Y$ so the restriction is injective on (ordinary) fundamental group. If the fundamental group becomes trivial remove $Y$ from $\operatorname{Ell}(\Gamma)$ and replace it by a tree. 
If $\varphi$ is not an immersion there is a vertex $v^{\prime}$ of $\Gamma^{\prime}$ and a connected component $v$ of $\varphi^{-1}\left(v^{\prime}\right)$ such that the induced map of fundamental groups of stars is not injective. The map extends to $\Gamma \cup D$, as in Figure 3, mapping the fourth side $h$ to a vertex. Now fold. If the fourth edge $h$ connects distinct elements of Ell( $\Gamma)$ the complexity decreases, and if the fourth edge $h$ connects a vertex $w$ to itself add $w \cup h$ to $\operatorname{Ell}(\Gamma)$. In this case $-\chi(\Gamma / \operatorname{Verts}(\Gamma))$ decreases. (Though $\mid$ Ell $\mid$ may increase.)

The last claim follows from the outermost arc argument.

Let $\Gamma$ be a relative graph. A basic rectangle in $\Gamma$ is a map $[0,1] \times[0,1] \rightarrow \Gamma$ such that $\{0\} \times[0,1]$ and $\{1\} \times[0,1]$ are mapped to 0 -cells meeting edges in $\Gamma$, and with image either completely contained in an element of $\operatorname{Ell}(\Gamma)$ or such that the sets $(0,1) \times\{y\}$ are mapped homeomorphically to interiors of edges of $\Gamma$. A rectangle in $\Gamma$ is either a rectangle of the second type or a map of a square $[0,3] \times[0,1] \rightarrow \Gamma$ such that $[0,1] \times[0,1] \rightarrow \Gamma$ and $[2,3] \times[0,1] \rightarrow \Gamma$ are basic rectangles of the second type and $[1,2] \times[0,1] \rightarrow \Gamma$ is a basic rectangle of the first type. If $R$ is a rectangle define $\partial^{L}(R)=\{0\} \times[0,1], \partial^{R}(R)=\{1\} \times[0,1]$, and $\partial^{+}(R)=[0,1] \times\{1\}$ and $\partial^{-}(R)=[0,1] \times\{0\}$.

An edge path in a relative graph is a morphism of a subdivided interval. An edge path which is also an immersion is reduced. Any edge path is homotopic, relative to its endpoints, to a reduced edge path, and any two reduced edge paths homotopic relative to endpoints are homotopic through reduced edge paths, i.e., they differ by reparametrization of the interval and a homotopy supported on subsegments mapping to elements of $\operatorname{Verts}(\Gamma)$. See Figure 4. A homotopy between two reduced edge paths is a concatenation of basic rectangles.

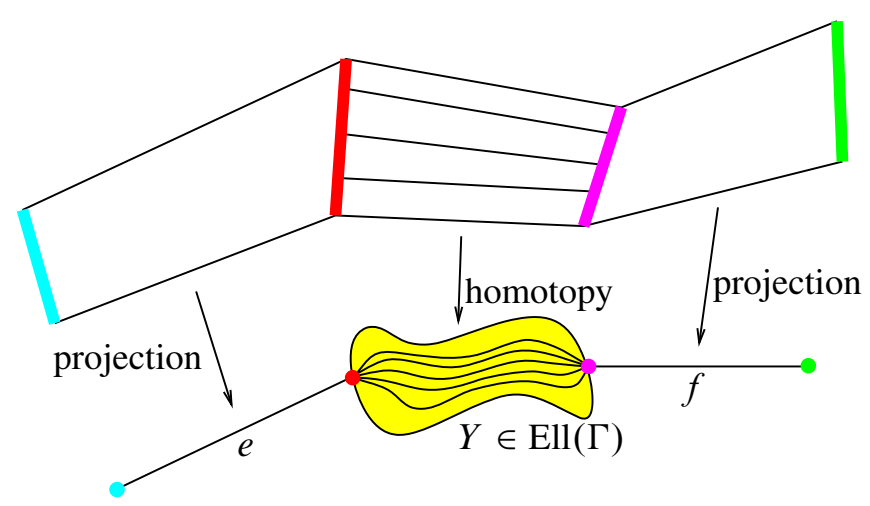

Figure 4. Homotopy between reduced edge paths is a concatenation of rectangles. Here are three basic rectangles whose concatenation is a rectangle.

The following lemma also follows from the innermost disc/outermost arc argument. 
Lemma 3.6. Let $\Gamma$ be a relative graph, let $p_{0}, p_{1}: S^{1} \rightarrow \Gamma$ be two homotopic reduced edge paths, and let $P: A \rightarrow \Gamma$ be a map of an annulus $A=S^{1} \times I$ such that $\left.P\right|_{S^{1} \times\{i\}}=p_{i}$. Then there is a map $Q: A \rightarrow \Gamma$ which restricts to $p_{0}$ and $p_{1}$ on the boundary and is a cycle of basic rectangles.

A map of an annulus as in the lemma is admissible. An admissible annulus is a homotopy between two cyclically reduced paths through cyclically reduced paths.

One important property of immersions of relative graphs is that if $p$ is a reduced edge path then the composition of $p$ and an immersion is also a reduced edge path. More generally, compositions of immersions are immersions.

3.2. Graphs of graphs II. The objective of this section is to prove Theorem 1.3, which we reduce to an analysis of graphs of graphs arising by adjoining roots to free groups.

For the remainder of this section, fix an inclusion $\eta: G \hookrightarrow H$ of finitely generated groups which lifts to an epimorphism $\pi: G^{\prime} \rightarrow H$. We may assume, without loss of generality, that for all $H^{\prime}$ such that if $G^{\prime} \rightarrow H$ factors though $H^{\prime}$ then $\chi_{\mathrm{fd}}\left(H^{\prime}\right)=\chi_{\mathrm{fd}}(H)$. By Lemma 3.5:

Lemma 3.7. Let $H^{\prime} \rightarrow H$ be as above. Then

$$
\chi_{\mathrm{fd}}\left(H^{\prime}\right) \geq \chi_{\mathrm{fd}}(H) .
$$

If a freely indecomposable free factor of $H^{\prime}$ has trivial image in $H$ then the inequality is strict.

We represent $\eta$ as an immersion $\varphi: \Gamma_{G} \rightarrow \Gamma_{H}$ and construct a space $X$, similar to a graph of graphs, with fundamental group $G\left[\sqrt[k]{\gamma_{i}}, \sqrt[l]{i} \sqrt{\delta_{j}}\right]$. This space has a nice map to $\Gamma_{H}$ representing $\pi$. Furthermore, by resolving, as in the case of a free group, $X$ may be given a graph of spaces structure "transverse" to the old one. After adjusting $X$ and analyzing the structure of vertex spaces we reduce the proof of Theorem 1.3 to Theorem 2.1.

Choose an relative graph $\Gamma_{H}$ with fundamental group $H$, and whose components $Y_{1}, \ldots, Y_{p} \in \operatorname{Ell}\left(\Gamma_{H}\right)$ have freely indecomposable fundamental groups. By Lemma 3.5 there is a relative graph $\Gamma_{G}$ with fundamental group $G$, and an immersion $\varphi: \Gamma_{G} \leftrightarrow \Gamma_{H}$ representing $\eta$. Choose, for each $\gamma_{i}, \delta_{j}$, immersions $\gamma_{i}: S^{1} \leftrightarrow \Gamma_{G}$, $\delta_{j}: S^{1} \leftrightarrow \Gamma_{G}$, representing the conjugacy classes $\left[\gamma_{i}\right],\left[\delta_{j}\right]$. Each immersion $\gamma_{i}: S^{1} \rightarrow \Gamma_{G}$ has image in the connected component of Ell $\left(\Gamma_{G}\right)$ representing $G_{i}$.

Let $M_{j}$ be the mapping cylinder of the $l_{j}$ fold cover $S_{j}^{1} \rightarrow S^{1}$, and let $r_{j}$ be the core of $M_{j}$. Now glue the $M_{j}$ along $S_{j}^{1}$ to $\Gamma_{G}$ using the immersions $\delta_{j}$ as attaching maps to form a space $X$. For each $r_{j}$, choose an immersion $\sqrt[l_{i}]{\delta_{j}}: r_{j} \rightarrow \Gamma_{H}$ representing the conjugacy class of $\sqrt[l]{\delta_{j}} \in H$. The map $\varphi: \Gamma_{G} \rightarrow \Gamma_{H}$ lifts to a continuous map $\pi: X \rightarrow \Gamma_{H}$ agreeing with the immersions $r_{j}$ and $\varphi$. Each mapping cylinder 
$M_{j}$ is a quotient space of an annulus $A_{j}$, and by Lemma 3.6 we may assume that $\pi$ induces admissible maps of annuli. Set $\Gamma(X)=\Gamma_{G} \sqcup \sqcup r_{j} ; \Gamma_{G}(X)$ plays the role that $\Gamma_{\infty}(X)$ does in the free case.

A relative graph $\Gamma$ is admissible if it is bipartite (elements of Verts $(\Gamma)$ can be divided into two classes such that each edge connects an element of one class to an element of the other class), all elements of one class of vertices are empty and valence two (the inessential vertices), and stars of elements of the other class of vertices (the essential vertices) embed. If $S$ is a star of an essential vertex then we give $S$ the structure of a relative graph with boundary by setting $\partial S=\overline{(\Gamma \backslash S)} \cap S$. An admissible relative graph is recovered by identifying boundary components of stars of essential vertices in pairs. Let $\mathcal{N}(\Gamma)$ be the collection of all stars of essential vertices.

If $\varphi: \Gamma \rightarrow \Gamma^{\prime}$ is an immersion of relative graphs and $\Gamma^{\prime}$ is admissible, $S \subset \Gamma^{\prime}$ a star of an essential vertex, then the connected components of $\varphi^{-1}(S)$ are (embedded!) stars of vertices of $\Gamma$.

Let $X$ be as above. Let $\mathcal{Z}$ be the collection of connected components of $\pi^{-1}(N)$, $N \in \mathcal{N}\left(\Gamma_{H}\right)$, and let $\mathscr{B}$ be the collection of connected components of the preimages of inessential vertices. Since each mapping cylinder induces an admissible map of the associated annulus, the elements of $\mathcal{B}$ are two-sided graphs contained in $X$. For each $B \in \mathscr{B}$ there are two embeddings of $B$ into elements of $\mathcal{Z}$. Since $\gamma_{i}$ is conjugate into some $G_{j}$ the associated mapping cylinder $M_{i}$ is contained in some $Z \in \mathcal{Z}$. The boundary of $Z \in Z$ is the set $B \cap Z$, and coincides with the set of points of $Z$ mapping to inessential vertices of $\Gamma_{H}$.

Each mapping cylinder is either completely contained in some $Z \in \mathcal{Z}$ or has nontrivial intersection with $\mathscr{B}$. If $M$ has nontrivial intersection with $\mathscr{B}$ then $r \cap Z$ (recall that $r$ is the core curve of $M$ ) is a collection of closed intervals, and the preimage $\mathscr{B}$ slices an annulus $A \in \mathcal{A}$ into rectangles.

The connected components of the intersections of the relative graph $\Gamma(X)$ and elements of $\mathcal{Z}$ are relative graphs $S$ with distinguished valence one vertices $S \cap \mathscr{B}$. Let $\delta(Z)$ be the collection of connected components of $\Gamma(X) \cap Z$. If $S \in S$ set $\partial S=S \cap \mathcal{B}$. For each $Z \in \mathcal{Z}$ let $\mathcal{R}(Z)$ be the rectangles contained in $Z$.

The boundary of each rectangle $R$ is composed of two types of arcs, $\partial^{ \pm} R=$ $R \cap \Gamma(X)$ and $\partial^{L \mid R} R=R \cap \mathscr{B}$. The former are horizontal boundary arcs and the latter are vertical boundary arcs. For each rectangle $R$ let $\varphi_{R}^{ \pm}: \partial^{ \pm} R \rightarrow S(R, \pm)$ be the attaching map for the horizontal/vertical boundary arc of $R$. Then $Z$ is the quotient space

$$
\delta(Z) \sqcup \mathcal{R}(Z) /\left(x \sim \varphi_{R}^{+}(x), y \sim \varphi_{R}^{-}(y)\right) .
$$

The boundary of $Z$ is the union of vertical boundary arcs of the rectangles comprising it, along with all valence one vertices of $\partial S(Z)$ not contained in some vertical boundary arc of a rectangle.

Let $N \in \mathcal{N}\left(\Gamma_{H}\right)$ be such that $Z$ is a connected component of $\varphi^{-1}(N)$. Let $\left\{b_{1}, \ldots, b_{k}\right\}$ be the valence one vertices comprising $\partial N$, with incident edges $e_{i} \subset N$ 


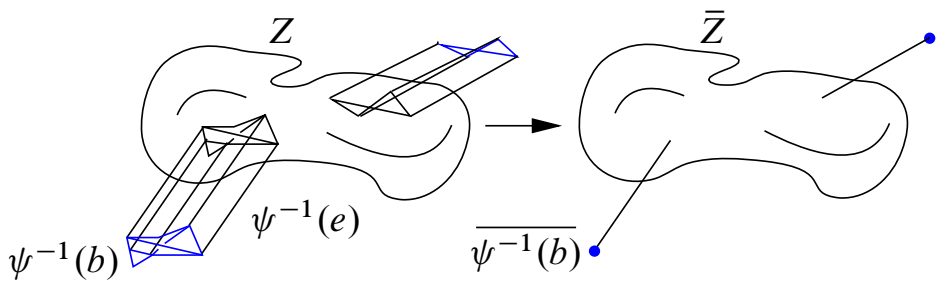

Figure 5. Crushing collars of boundary components.

so that $\tau\left(e_{i}\right)=b_{i}$. If $\psi: Z \rightarrow N$ is the restriction of $\pi$ then $\psi^{-1}\left(e_{i}\right)$ is a collar neighborhood of $\psi^{-1}\left(b_{i}\right) \in \mathscr{B}$, a boundary component of $Z$. The restriction $\psi$ factors through the map which projects each collar onto the I factor. Call the resulting quotient space $\bar{Z}$.

The map $\pi: X \rightarrow \Gamma_{H}$ factors through the graph of spaces $\Gamma_{K}$ obtained by assembling the collection $\{\bar{Z} \mid Z \in Z$ Z along images of boundary components: if $B \subset Z_{1}, Z_{2}$, then identify the images $\bar{B} \in \bar{Z}_{1}$ and $\bar{B} \in \bar{Z}_{2}$. Let $K=\pi_{1}\left(\Gamma_{K}\right)$.

Lemma 3.8. Each space $\bar{Z}$ has freely indecomposable or trivial fundamental group.

Proof. If some $\bar{Z}$ had freely decomposable fundamental group then $\chi_{\mathrm{fd}}(K)>\chi_{\mathrm{fd}}(H)$, contradicting our choice of $H$.

If $q_{H}=q_{G}$, then no $S \in \delta(Z)$ has fundamental group with nontrivial free part. In particular, elements of $\delta(Z)$ are all relative trees. Let $G=G(Z)$ be the graph with vertex set $\delta(Z)$ and edge set $\mathcal{R}(Z)$. The endpoints of an edge $R$ are the boundary components $\partial^{ \pm} R$, and an endpoint $\partial^{ \pm} R$ is attached to $S$ if the image of $\varphi_{R}^{ \pm}$is contained in $S$. Let $T$ be a maximal tree in $G$ and let $Z_{T}$ be the space obtained by restricting to $T$.

Since no $S \in S(Z)$ has nontrivial free part and $G$ embeds in $H$, the components $Z \in \mathcal{Z}$ fall into three classes:

(1) $Z_{1}=\left\{Z \mid \pi_{1}(\bar{Z})\right.$ is trivial $\}$.

(2) $Z_{2}=\left\{Z \mid \pi_{1}(\bar{Z})\right.$ is nontrivial and $Z$ contains no $\left.Y \in \operatorname{Ell}\left(\Gamma_{G}\right)\right\}$.

(3) $Z_{3}=\left\{Z \mid \pi_{1}(\bar{Z})\right.$ is nontrivial and $Z$ contains some $\left.Y \in \operatorname{Ell}\left(\Gamma_{G}\right)\right\}$.

Since $G \hookrightarrow H$, if $Z \in Z_{1}$, then $Z$ contains no $Y \in \operatorname{Ell}\left(\Gamma_{G}\right)$.

We again reduce the computation $\chi_{\mathrm{fd}}(H) \leq \chi_{\mathrm{fd}}(G)$ to a version of the combinatorial Gauss-Bonnet Theorem.

Definition 3.9 ( $q$-excess/deficiency). For each $Z$, let

$$
\Delta_{q}^{-}(Z)=\frac{1}{2}\left(\# \partial Z_{T}-\# \partial \bar{Z}\right),
$$


for $Z \in Z_{3}$ let

$$
\Delta_{q}^{+}(Z)=\frac{1}{2} b_{1}\left(\partial Z_{T}\right)
$$

and for $Z \in Z_{1} \cup Z_{2}$ set

$$
\Delta_{q}^{+}(Z)=0 .
$$

If $S$ is a relative graph with no loops (no contribution to $q_{G}$ ), set $\kappa(S, \partial S)=$ $\frac{1}{2} \# \partial S-1$,

Definition 3.10 ( $p$-excess/deficiency). If $Z \in Z_{1}$ then define $\Delta_{p}^{+}(Z)=\Delta_{p}^{-}(Z)=0$. If $Z \in Z_{2}$ then define $\Delta_{p}^{+}(Z)=1$ and $\Delta_{p}^{-}(Z)=0$. If $Z \in \mathcal{Z}_{3}$ and $Y_{0}, \ldots, Y_{k}$ are the components of $\operatorname{Ell}\left(\Gamma_{G}\right)$ contained in $Z$, then define $\Delta_{p}^{-}(Z)=k$ and $\Delta_{p}^{+}(Z)=0$.

\section{Lemma 3.11.}

$$
\kappa(\bar{Z}, \partial \bar{Z})-\sum_{S \in S(Z)} \kappa(S, \partial S)=\Delta_{q}^{+}(Z)-\Delta_{q}^{-}(Z) .
$$

Proof. We need to show that

$$
\frac{1}{2} \# \partial Z_{T}-1=\sum_{S \in \mathcal{S}(Z)}\left(\frac{1}{2} \# \partial S-1\right)+\Delta_{q}^{+}(Z) .
$$

Let $T \subset G(Z)$ be a maximal tree, and let $S_{1}, \ldots, S_{k}$ be an enumeration of $\delta(Z)$ such that $S_{i+1}$ is connected to $S_{0} \cup R_{0} \cup S_{1} \cup R_{1} \ldots S_{i}$ by an edge $R_{i} \subset T$. Assume that $R_{i}$ is oriented so that $\partial^{+} R_{i}$ is attached to $S_{i+1}$. Let $Z_{i}$ be the union of $S_{1}, \ldots, S_{i}$ and $R_{1}, \ldots, R_{i-1}$.

The boundary of $\partial^{+/-} R_{i}$ consists of two components. Suppose that for at least one of + or - , the image $\partial \partial^{+-} R_{i}$ is contained in two distinct boundary components of at least one of $Z_{i}$ or $S_{i+1}$. If this is the case then

$$
\begin{aligned}
\kappa\left(Z_{i+1}, \partial Z_{i+1}\right) & =\frac{1}{2} \# \partial Z_{i+1}-1 \\
& =\frac{1}{2}\left(\# \partial Z_{i}+\# \partial S_{i+1}-2\right)-1 \\
& =\kappa\left(Z_{i}, \partial Z_{i}\right)+\kappa\left(S_{i+1}, \partial S_{i+1}\right) .
\end{aligned}
$$

If both $\partial \partial^{+} R_{i}$ and $\partial \partial^{-} R_{i}$ have image in the same boundary component of $S_{i+1}$, $Z_{i}$, respectively, then $Z_{i}$ contains at least $b_{1}\left(\partial Z_{i}\right)+1$ elements of Ell $\left(\Gamma_{G}\right)$ and $S_{i+1}$ must contain a new element of $\operatorname{Ell}\left(\Gamma_{G}\right)$. We then have

$$
\begin{aligned}
\kappa\left(Z_{i+1}, \partial Z_{i+1}\right) & =\frac{1}{2} \# \partial Z_{i+1}-1 \\
& =\frac{1}{2}\left(\# \partial Z_{i}+\# \partial S_{i+1}-1\right)-1 \\
& =\kappa\left(Z_{i}, \partial Z_{i}\right)+\kappa\left(S_{i+1}, \partial S_{i+1}\right)+\frac{1}{2} .
\end{aligned}
$$


Each such rectangle makes a contribution of +1 to $b_{1}\left(\partial Z_{T}\right),+1 / 2$ to $\Delta_{q}^{+}$, and only such rectangles make such contributions, thus

$$
\kappa\left(Z_{T}, \partial Z_{T}\right)=\sum_{S \in \mathcal{S}(Z)} \kappa(S, \partial S)+\Delta_{q}^{+}(Z) .
$$

Lemma 3.12. If $Z \in Z_{3}$ then $\Delta_{p}^{-}(Z) \geq b_{1}\left(\partial Z_{T}\right)=2 \Delta_{q}^{+}(Z)$.

Consider Figure 6. The tree $T$ in this example is a tripod, there is one boundary component of $Z_{T}$, and $\Delta_{p}^{-}=2$. A similar example with only two elements in $S(Z)$ would have $\Delta_{p}^{-}=1$. We argue that each independent loop in $\partial Z_{T}$ must contribute at least 1 to $\Delta_{p}^{-}$.

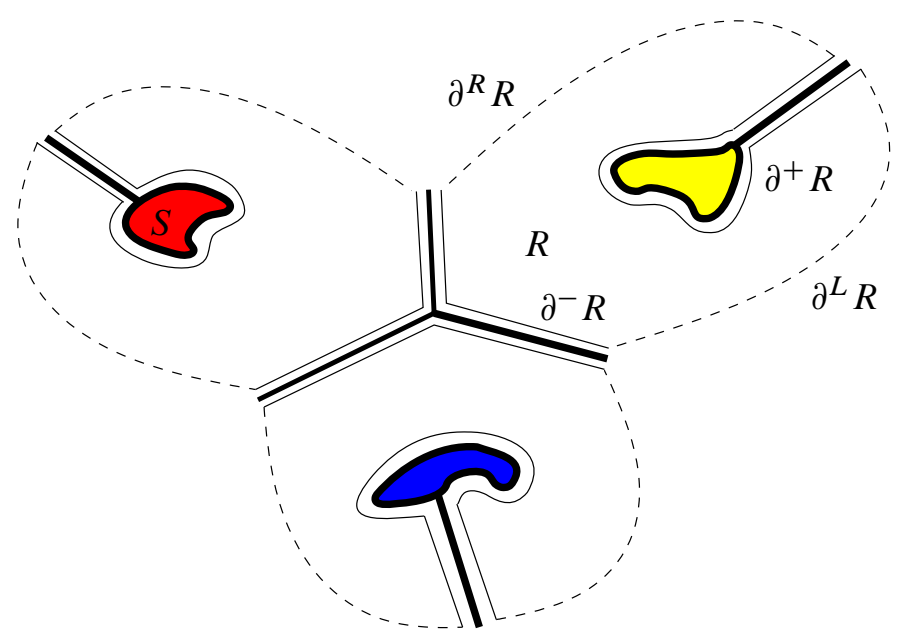

Figure 6. Illustration for Lemma 3.12. If $b_{1}\left(\partial Z_{T}\right) \neq 0$ then $Z_{T}$ contains too many elements of $\operatorname{Ell}\left(\Gamma_{G}\right)$, which will force $\chi_{\mathrm{fd}}$ to decrease.

Proof of Lemma 3.12. Argue as in Lemma 3.11. If $\partial \partial^{ \pm} R$ maps to a single boundary component of $S \in \delta(Z)$ then, since $\partial^{ \pm} R \rightarrow S$ is an immersion, $S$ must have nontrivial fundamental group, and since the free part of $S$ is trivial, it must contain some element $Y$ of $\operatorname{Ell}\left(\Gamma_{G}\right)$.

Choose the exhaustion of $T$ so that $S_{0}$ has an incident edge $R, \partial^{-} R \rightarrow S_{0}$ such that $\partial \partial^{-} R$ maps to a single boundary vertex of $S_{0}$. Let $R_{i_{1}}, \ldots, R_{i_{b_{1}\left(\partial Z_{T}\right)}}$ be the rectangles such that $\partial \partial^{+} R_{i_{j}}$ maps to a single boundary component of $S_{i_{j}+1}$. Then each $S_{i_{j}+1}$ contains some element $Y_{j} \in \operatorname{Ell}\left(\Gamma_{G}\right)$. Since the $S_{i_{j}}$ are distinct, $Z$ contains at least $b_{1}\left(\partial Z_{T}\right)+1$ elements of $\operatorname{Ell}\left(\Gamma_{G}\right)$, i.e., $\Delta_{p}^{-}(Z) \geq b_{1}\left(\partial Z_{T}\right)$.

Lemma 3.13. If $Z \in Z_{2}$ then $\Delta_{q}^{-}(Z) \geq \frac{1}{2}$. If equality holds then $\pi_{1}(\bar{Z}) \cong \mathbb{Z}_{2}$. 
Proof. Suppose that $\Delta_{q}^{-}(Z)>0$. Then $\Delta_{q}^{-}(Z) \geq \frac{1}{2}$. Suppose that $\Delta_{q}^{-}(Z)$ is $\frac{1}{2}$. Let $Z_{T}$ be as before, and attach a rectangle $R$ to $Z_{T}$ such that $\# \partial Z_{T} \cup R=\# \partial Z_{T}-1$. Let $R_{1}, \ldots, R_{n}, S^{ \pm}$be a path in $T$ connecting the endpoints of $R$. Then $R$ and the $R_{i}$ must form a Möbius strip, as in Figure 7. Adding rectangles while maintaining $\Delta_{q}^{-}(Z)=\frac{1}{2}$ does not change the fundamental group of $\bar{Z}$, which is clearly $\mathbb{Z}_{2}$.

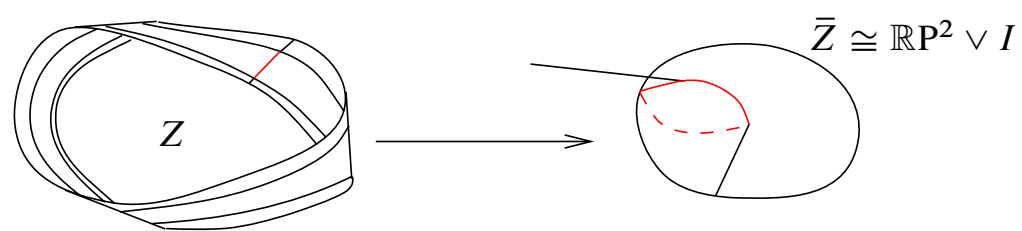

U

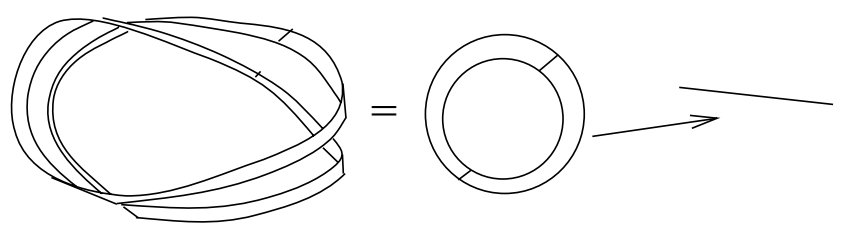

Figure 7. Collapsing a collar neighborhood of a Möbius strip to build a projective plane.

Lemma 3.14. $\chi_{\mathrm{fd}}(H) \leq \chi_{\mathrm{fd}}(G)$. If equality holds then for all $Z \in \mathcal{Z}_{2}, \Delta_{q}^{-}(Z)=\frac{1}{2}$. In particular, every such $\bar{Z}$ has fundamental group $\mathbb{Z}_{2}$.

If $H$ has no $\mathbb{Z}_{2}$ free factors and equality holds then $\mathbb{Z}_{2}$ is empty, all members of $Z_{3}$ contain exactly one element of Ell $\left(\Gamma_{G}\right)$, and $\Delta_{p \mid q}^{ \pm}(Z)=0$ for all $Z$.

Proof. By Gauss-Bonnet,

$$
\sum_{Z \in Z} \sum_{S \in \mathcal{S}(Z)} \kappa(S, \partial S)=q_{G}-1
$$

and, since the maps $\bar{Z} \rightarrow H$ do not factor through free products, again by GaussBonnet,

$$
\sum_{Z \in Z} \kappa(\bar{Z}, \partial \bar{Z})=q_{H}-1 .
$$

By homology, $q_{H}-1 \leq q_{G}-1$, and if $q_{H}-1=q_{G}-1$, then by Lemma 3.11,

$$
\sum_{Z \in Z_{3}} \Delta_{q}^{+}(Z)=\sum_{Z \in Z} \Delta_{q}^{-}(Z),
$$


and we compute

$$
\begin{array}{rlr}
p_{G} & =\left|Z_{3}\right|+\sum_{Z \in Z_{3}} \Delta_{p}^{-}(Z) & \text { (Definition) } \\
& \geq\left|Z_{3}\right|+2 \sum_{Z \in Z_{3}} \Delta_{q}^{+}(Z) & \text { (Lemma 3.12) } \\
& =\left|Z_{3}\right|+2 \sum_{Z \in Z} \Delta_{q}^{-}(Z) & \text { (Lemma 3.11) } \\
& =\left|Z_{3}\right|+2 \sum_{Z \in Z_{2}} \Delta_{q}^{-}(Z) & \text { (obvious) } \\
& \geq\left|Z_{3}\right|+\left|Z_{2}\right| & \text { (Lemma 3.13) } \\
& =p_{H} . &
\end{array}
$$

Each $Z \in Z_{3}$ is a union of stars of vertices of $\Gamma(X)$, rectangles from mapping cylinders representing roots adjoined to elements $\delta_{j}$, and mapping cylinders representing roots adjoined to elements $\gamma_{i}$ which are conjugate into freely indecomposable free factors of $G$. Let $Y(Z)$ be the only element of $\operatorname{Ell}\left(\Gamma_{G}\right)$ contained in $Z$, let $S_{0}(Z)$ be the element of $S(Z)$ containing $Y(G)$, and let $\mathcal{M}(Z)$ be the collection of mapping cylinders attached to $Y(Z)$. Let $\mathcal{R}(Z)$ again stand for the rectangles contained in $Z$. For each attaching map $p: \partial^{ \pm} R \rightarrow(S, \partial S)$ we choose a fixed reduced edge path $\hat{p}:\left(I_{p}, \partial I_{p} \rightarrow(S, \partial S)\right.$ such that if $[p]=\left[p^{\prime}\right] \in \pi_{1}((S, \partial S))$ then there are maps $q: \partial^{ \pm} R \rightarrow(I, \partial I)$ and $q^{\prime}: \partial^{ \pm} R^{\prime} \rightarrow(I, \partial I)$ such that $\hat{p} q=p$ and $\hat{p} q^{\prime}=p^{\prime}$.

Define an equivalence relation $\sim$ on the collection of rectangles $R$ by $R \sim R^{\prime}$ if $\left[\partial^{ \pm} R \rightarrow(S, \partial S)\right]=\left[\partial^{ \pm} R^{\prime} \rightarrow(S, \partial S)\right]$ and $R$ and $R^{\prime}$ are not separated by a cutpoint in $G(Z)$.

The following is the analog of Lemma 2.4 for the vertex spaces $Z \in Z_{3}$.

Lemma 3.15. Let $Z \in Z_{3}$ and suppose that

- $S_{i} \in S(Z)$ is a tree unless $i=0$,

- the fundamental group of $S_{0}$ embeds under the map $Z \rightarrow Z / \partial Z$.

Then

- If $S \in S$ is not a cutpoint in $G(Z)$ then there is only one homotopy class of attaching map associated to $S$.

- The $\sim$ equivalence classes are exactly the maximal cut-point-free subgraphs of $G(Z)$.

Proof. Consider a chain of rectangles $R_{0}, \ldots, R_{n-1}$ forming an embedded cycle in $G(Z)$, and orient the edges of $G(Z)$ so that, for each $i, \partial^{+} R_{i}$ and $\partial^{-} R_{i+1}$ are mapped to the same vertex space $S_{i}$. If $\partial^{+} R_{i}$ and $\partial^{-} R_{i+1}$ are attached to the same $S_{j_{i}}, j_{i} \neq 0$, then the maps must agree up to reversal of orientation since $\Delta_{q}^{-}(Z)=0$. 


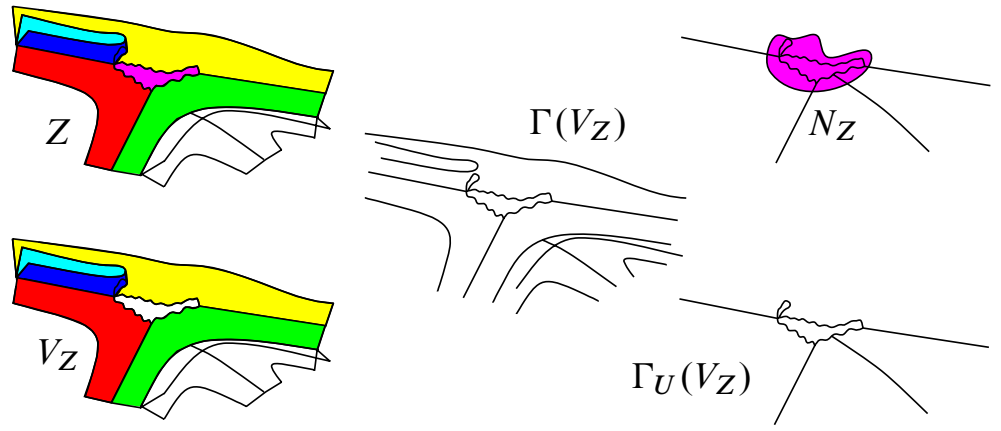

Figure $8 . V_{Z}$ and all the rest.

Orient all rectangles so that $\left[\partial^{+} R_{i} \rightarrow S_{j_{i}}\right]=\left[\partial^{-} R_{i+1} \rightarrow S_{j_{i}}\right]$ agree. Then the concatenation of rectangles, in $Z / \partial Z$ represents a homotopy between $\left[\partial^{+} R_{0} \rightarrow S_{j_{0}}\right]$ and $\left[\partial^{-} R_{n-1} \rightarrow S_{j_{0}}\right]$. Since $\pi_{1}\left(S_{0}\right)$ embeds under $Z \rightarrow Z / \partial Z$, the attaching maps are equal.

If $S$ is not a cutpoint then every pair of edges adjacent to $S$ is contained in an embedded cycle in $G(Z)$. Any maximal cutpoint-free subgraph contains an embedded loop passing through any pair of edges, hence the second bullet holds as well.

Let $Z \in Z_{3}$ be as above. Let $B_{i}$ be the maximal cut-point-free subgraphs of $G(Z)$. For each class of attaching map $p: \partial^{ \pm} R \rightarrow(S, \partial S)$ let $I_{p}$ be an interval as before, and let $Z_{i}$ be the graph of spaces obtained by replacing each $S \in B_{i}$ by $I_{p}$. Let $p_{1}, \ldots, p_{k}$ be the attaching maps for rectangles adjacent to $S_{0}=\operatorname{St}(Y(Z))$, where $Y(Z)$ is the element of $\operatorname{Ell}\left(\Gamma_{G}\right)$ contained in $S_{0}$. Let $e_{1}, \ldots, e_{l}$ be the oriented edges of $X$ terminating in $Y$. Each path $p_{i}$ is a concatenation of paths $e_{a(i)} r_{i} \bar{e}_{b(i)}$. Construct a new graph $S_{0}^{\prime}$ by identifying the initial and terminal segments of $I_{p_{i}}$ mapping to the same edges of $\operatorname{St}(Y)$. We recover $S_{0}$ by attaching $S_{0}^{\prime}$ to $Y(Z)$ by gluing the subgraph $D_{Z}$ of $S_{0}^{\prime}$ associated to the middle segments of the $I_{p}$ along the paths $r_{i}$. There is a natural map $I_{p} \rightarrow S_{0}^{\prime}$. Define a related space $V_{Z}$ by taking $\mathcal{S}\left(V_{Z}\right)=\left\{S_{0}^{\prime}, S_{1}, \ldots, S_{n}\right\}$ as vertex spaces and attaching the rectangles from $\mathcal{R}(Z)$ to $S\left(V_{Z}\right)$ with the induced attaching maps. Then $Z$ is recovered by gluing the image of $D_{Z}$ in $V_{Z}$ to $Y$ along the map $\psi_{Z}: D_{Z} \rightarrow Y(Z)$ induced by the $r_{i}$ and reattaching the mapping cylinders in $\mathcal{M}(Z)$ to $Y$.

Each $Z_{i}$ is homeomorphic to a product $B_{i} \times I$. Give $B_{i} \times I$ the foliation induced by projection to the $I$ coordinate, and give each $S \in S\left(V_{Z}\right)$ the foliation whose leaves are simply the points of $S$. Then define $F(Z)$ to be the foliation on $V_{Z}$ induced by the foliations on $B_{i} \times I$ and $S$. Define $\Gamma_{U}\left(V_{Z}\right)$ to be the leaf space of $F(Z)$, and denote the quotient map by $\pi_{Z}$. Let $\partial \Gamma_{U}\left(V_{Z}\right)$ be the image of the boundary of $Z$. The following lemma is obvious from the construction. 
If $\Gamma$ is a graph with boundary $\partial \Gamma$, define

$$
\kappa(\Gamma, \partial \Gamma)=-\chi(\Gamma)+\frac{1}{2} \# \partial \Gamma .
$$

Lemma 3.16. The following hold for $\Gamma_{U}\left(V_{Z}\right)$ :

- $\Gamma_{U}\left(V_{Z}\right)$ is a graph.

- $\kappa\left(\Gamma_{U}\left(V_{Z}\right), \partial \Gamma_{U}\left(V_{Z}\right)\right)=\sum_{S \in S\left(V_{Z}\right)} \kappa(S, \partial S)$

- Each boundary component of $\Gamma_{U}\left(V_{Z}\right)$ is the image of exactly one boundary component of $Z^{\prime}$.

- Point preimages under $\pi_{Z}$ are connected and the natural maps $(S, \partial S) \rightarrow$ $\left(\Gamma_{U}\left(V_{Z}\right), \partial \Gamma_{U}\left(V_{Z}\right)\right)$ are immersions.

The following extension property also holds. Let $f: Z \rightarrow A$ be a continuous map to an aspherical space $A$, mapping each boundary component of $Z$ to a point. Then there exists $f^{\prime}: \Gamma_{U}\left(V_{Z}\right) \rightarrow A$ such that $f^{\prime} \circ \pi_{Z}$ and $f$ are homotopic rel $\partial Z$. In particular, they induce the same map on $\pi_{1}(Z, \partial Z)$.

Let $N_{Z}$ be the space obtained by attaching $\Gamma_{U}\left(V_{Z}\right)$ and all mapping cylinders in $\mathcal{M}(Z)$ to $Y(Z)$, and let $\mathcal{N}$ be the collection of all such $N_{Z}$. The space $X$ is the pushout of the diagram $\mathscr{B} \hookrightarrow \mathcal{Z}$. The map $\mathcal{B} \hookrightarrow \mathcal{Z}$ factors through the natural map $\mathscr{B} \hookrightarrow \mathcal{V}$, and the map $X \rightarrow \Gamma_{H}$ factors through the map crushing all elements of $\mathscr{B}$ to points. By Lemma $3.16 \overline{\mathcal{B}} \rightarrow \overline{\mathcal{Z}}$ factors through the natural map $\overline{\mathcal{B}} \rightarrow \mathcal{N}$, and by Van Kampen, $X \rightarrow \Gamma_{H}$ then factors, up to homotopy, through the pushout of $\bar{B} \rightarrow \mathcal{N}$.

Define a new graph of graphs $X^{\prime}$ as the pushout of $\mathscr{B} \rightarrow \mathcal{V}$. The underlying graph of $X^{\prime}$ is obtained as the pushout $\overline{\mathscr{B}} \rightarrow \bigsqcup \Gamma_{U}\left(V_{Z}\right)$. Then $\Gamma\left(X^{\prime}\right)$ is the pushout $\mathscr{B}^{0} \rightarrow \bigsqcup S\left(V_{Z}\right)$.

It is clear that $\Gamma\left(X^{\prime}\right)$ and $\Gamma_{U}\left(X^{\prime}\right)$ are graphs, and, since point preimages of $V_{Z} \rightarrow \Gamma_{U}\left(V_{Z}\right)$ are connected, $\pi_{1}\left(X^{\prime}\right) \rightarrow \pi_{1}\left(\Gamma_{U}\left(X^{\prime}\right)\right)$ is onto. The attaching maps $M \rightarrow \Gamma_{G}$ factor through the inclusion $\Gamma_{\infty}\left(X^{\prime}\right) \hookrightarrow X^{\prime}$, and we have $X=$ $\Gamma_{\infty}(X) \bigcup_{\delta_{j}}\left\{M_{j}\right\}$.

We recover $X$ in the same way as we recover $Z$ : by gluing each $D_{Z} \subset \Gamma\left(V_{Z}\right)$ to $Y(Z)$ via the map $\psi_{Y}$. Since each $\Gamma\left(V_{Z}\right)$ immerses in $\Gamma_{Z}$ under $\pi_{Z} \Gamma(X) \rightarrow \Gamma_{U}(X)$ is an immersion.

Lemma 3.17. $\chi\left(\Gamma\left(X^{\prime}\right)\right)=\chi\left(\Gamma_{U}\left(X^{\prime}\right)\right)$.

Proof. The proof is the straightforward computation. By Lemma 3.16 and definition,

$$
\begin{aligned}
-\chi\left(\Gamma\left(X^{\prime}\right)\right) & =\sum_{Z \in \mathcal{Z}} \sum_{S \in \mathcal{S}\left(V_{Z}\right)} \kappa(S, \partial S) \\
& =\sum_{Z \in \mathcal{Z}} \kappa\left(\Gamma_{U}\left(V_{Z}\right), \partial \Gamma_{U}\left(V_{Z}\right)\right)=-\chi\left(\Gamma_{U}\left(X^{\prime}\right)\right) .
\end{aligned}
$$


The images of the $D_{Z}$ are disjoint under the map $X^{\prime} \rightarrow \Gamma_{U}\left(X^{\prime}\right)$. We now prove Theorem 1.3.

Proof of Theorem 1.3. It suffices to show that $G$ splits as $G^{\prime} *\left\langle\delta_{1}^{d_{1}}\right\rangle * \cdots *\left\langle\delta_{m}^{d_{m}}\right\rangle$. By Theorem 2.1 and connectivity of point preimages under $V_{Z} \rightarrow \Gamma_{U}\left(V_{Z}\right)$, edge spaces of $X^{\prime}$ are trees. Since the images of the $D_{Z}$ are disjoint under the map $X^{\prime} \rightarrow \Gamma_{U}\left(X^{\prime}\right)$ and the $\delta_{j}$ are not supported on $D_{Z}$, there is an edge of $\Gamma_{\infty}\left(X^{\prime}\right) \backslash \cup D_{Z}$ crossed by exactly one $\delta_{j}$, and $\delta_{j}$ is therefore conjugate to a primitive element of $G$ relative to $G_{i}$ and conjugates of the remaining $\delta_{j^{\prime}}, j^{\prime} \neq j$. Removing this edge from $\Gamma_{\infty}\left(X^{\prime}\right)$, along with the core curve of the mapping cylinder corresponding to $\delta_{j}$ and all rectangles adjacent to the core curve gives a graph of spaces of the same form.

Remark 3.18. Simply knowing that some $\delta_{j}$ is a primitive element of $\pi_{1}(\Gamma(X))$ is not sufficient to imply that $\left\langle\delta_{j}\right\rangle$ is a free factor of $G$, thus Theorem 1.3, even in the case $m=1$, cannot be deduced from Baumslag's theorem.

\section{References}

[1] G. Baumslag, Residual nilpotence and relations in free groups. J. Algebra 2 (1965), 271-282. Zbl 0131.02201 MR 0179239

[2] M. J. Dunwoody, Folding sequences. In The Epstein birthday schrift, Geom. Topol. Monogr. 1, University of Warwick, Institute of Mathematics, Coventry 1998, 139-158. Zbl 0927.20013 MR 1668347

[3] L. Louder and D. B. McReynolds, Graphs of subgroups of free groups. Algebr. Geom. Topol. 9 (2009), 327-335. Zbl 1185.20026 MR 2482080

[4] R. C. Lyndon, The equation $a^{2} b^{2}=c^{2}$ in free groups. Michigan Math. J 6 (1959), 89-95. Zbl 0084.02803 MR 0103218

[5] R. C. Lyndon and M. P. Schützenberger, The equation $a^{M}=b^{N} c^{P}$ in a free group. Michigan Math. J. 9 (1962), 289-298. Zbl 0106.02204 MR 0162838

[6] E. Schenkman, The equation $a^{n} b^{n}=c^{n}$ in a free group. Ann. of Math. (2) 70 (1959), 562-564. Zbl 0090.24501 MR 0104723

[7] G. P. Scott, Finitely generated 3-manifold groups are finitely presented. J. London Math. Soc. (2) 6 (1973), 437-440. Zbl 0254.57003 MR 0380763

[8] A. Shenitzer, Decomposition of a group with a single defining relation into a free product. Proc. Amer. Math. Soc. 6 (1955), 273-279. Zbl 0064.02204 MR 0069174

[9] J. R. Stallings, A topological proof of Grushko's theorem on free products. Math. Z. 90 (1965), 1-8. Zbl 0135.04603 MR 0188284

[10] J. R. Stallings, Topology of finite graphs. Invent. Math. 71 (1983), 551-565. Zbl 0521.20013 MR 695906

[11] G. A. Swarup, Delzant's variation on Scott complexity. Preprint 2004. arXiv:math/0401308 
[12] R. Weidmann, The Nielsen method for groups acting on trees. Proc. London Math. Soc. (3) 85 (2002), 93-118. Zbl 1018.20020 MR 1901370

Received May 17, 2010; revised September 3, 2011

L. Louder, Mathematics Institute, Zeeman Building, University of Warwick, Coventry CV4 7AL, United Kingdom

E-mail: L.Louder@warwick.ac.uk 